First received $3^{\text {rd }}$ October 2007. Revised version received and accepted $2^{\text {nd }}$ February 2008.

\title{
Symptom Awareness and Cancer Prevention: Exploratory Findings from an At-risk Population
}

\author{
Douglas Eadie and Susan MacAskill
}

\author{
Author details \\ Douglas Eadie (author for correspondence) \\ Senior Researcher \\ Susan MacAskill \\ Senior Researcher \\ Institute for Social Marketing \\ University of Stirling and The Open University \\ Stirling \\ FK9 4LA \\ Tel: $+44(0) 1786466459$ \\ Fax: +44 (0)1786 466449 \\ Emails: douglas.eadie@stir.ac.uk \& susan.macaskill@stir.ac.uk
}

Keywords: cancer, patient education, early detection, self-referral, symptom awareness Paper category: Research Paper 


\begin{abstract}
$\underline{\text { Abstract }}$
Purpose: Secondary prevention programmes have traditionally employed mass screening approaches to assess for asymptomatic signs of cancer. It has been suggested that early detection strategies involving public education and self-referral may prove more costeffective with low risk populations for cancers with symptomatic presentation. This study, which was commissioned to inform the development of a cancer awareness campaign, examines public perceptions of the early detection of cancer, the psycho-social barriers to self-referral amongst a key at-risk population and the implications for patient education.
\end{abstract}

Methodology: An exploratory study using qualitative focus groups with an at-risk population of older people living in deprived communities in west-central Scotland.

Findings: The findings reveal broad support for initiatives designed to raise symptom awareness. However fear of cancer can lead to apparently irrational responses to symptoms and subsequent delay, particularly amongst men who are less likely to seek support from lay networks.

Implications: Early detection messages need to take account tone and symptom description to allay fears and ensure appropriate presentation. Consideration also needs to be given to the role played by lay and professional opinion formers to informing and supporting patients decision to present with suspicious symptoms, particularly when targeting harder to reach patients of men, older people, and people living in deprived communities.

\title{
Introduction
}

Cancer incidence in the UK has increased from the 1970s (Quinn et al. 2005) and is now the most common cause of death for both men and women. Around a third of all people in the UK will develop cancer, with the majority of cancers occurring later in life - $64 \%$ of cancers occur in people aged 65 or over. Smoking tobacco, along with drinking alcohol and poor diet have been identified as risk factors for a wide range of cancers and helps explain higher rates for some cancers in areas of material deprivation. One such area is the central belt of Scotland, where incidence and mortality rates for cancer are $15 \%$ above the UK average.

Given these health inequalities and in recognition that early detection needs to form a key component of any comprehensive cancer control framework (CDCP 2002), the five local health boards in west-central Scotland formed a coalition to develop a public education campaign promoting awareness of cancer symptoms and encouraging self-referral to a local primary health care professional. This paper describes some key results to emerge from exploratory research conducted with the campaigns target group of older people at 
elevated risk of cancer which may be of interest to others developing similar work in the area of cancer symptom awareness. The study was based on the principal of evidencebased practice (Truman 2000) and was designed to provide first-hand insights into the target group's attitudes towards cancer detection and the factors which facilitate and hinder self-referral with suspicious symptoms.

Whilst the UK has witnessed a growth in the incidence of cancer in recent years, mortality rates for the disease have decreased over the same period. This has been attributed to a variety of factors including, better targeting of those at elevated risk, development of screening tests with improved predictive values, timely diagnosis and the provision of more effective treatments. An important concept underpinning much of this work has been secondary prevention, which is based on the premise that the earlier cancer is diagnosed the better the outcomes.

In the UK, like most other developed countries, the second half of the last century saw major advances in the development of mass screening as the main approach to secondary prevention. However, over the same period others have also highlighted the importance of encouraging individuals to seek earlier consultation with a doctor when they experience early warning signs of cancer (e.g. Box 1984). It is argued that self-referral approaches are preferable to mass screening of unselected populations where there is a scarcity of resources (Braveman and Tarimo 1996) and when targeting populations at relatively low risk (Bloom 1994). Self-referral approaches have been found to be particularly effective with patient populations who are directly effected by the disease and are therefore receptive to preventive messages (e.g. Duyn et al. 2002).

It is known that delay in treatment can affect health outcome and that patient delay in presenting to a GP is the main factor contributing to these outcomes (Anderson and Cacioppo 1995, Ristvedt and Trinkaus 2005). The value of public education programmes promoting symptom awareness is therefore dependent upon their ability to prompt the individual to self-refer earlier than they might otherwise and within the 'time window' when the cancer can be cured (Bloom 1994). There is also a growing body of evidence which indicates that those living in disadvantaged circumstances are more reluctant to engage in help-seeking behaviour (e.g. Eiser 2000). Given the apparent value of promoting symptom awareness this study sought to understand the factors that were influential in delaying self-referral amongst a socially disadvantaged at-risk population in west-central Scotland.

\section{Methods}

To provide the necessary flexibility required to understand the range of behavioural issues under investigation qualitative focus groups were employed, a method other studies have found useful for examining cancer prevention issues (e.g. Wolf et al. 2001, Beeker et al. 2000). 
A total of eight focus groups were conducted with a mean of seven participants per group $(n=57)$. Two experienced researchers moderated the discussions, one male and one female, and in three of the focus groups moderation was observed to ensure consistency across the study. In addition, a discussion guide was devised to assist group moderators cover the topics relevant to the research. Topics were not explored in a prescriptive manner. Instead, participants were given the freedom to express their own feelings and views as part of an open and free-ranging discussion. This flexible format enabled the researcher to develop original lines of enquiry, allowing salient topics and behavioural insights to emerge. Table 1 lists the main discussion themes.

\section{TABLE 1 ABOUT HERE}

Each focus group lasted approximately one and quarter hours and was held in a neutral venue such as a community centre or local hall. With the participants' consent, the discussion was recorded on voice-tape, and the tapes transcribed in full for thematic analysis. Transcripts were organised using a thematic framework based on topics specified in the discussion guide and emerging themes identified through a process of familiarisation with transcript texts. Coding of the voice-transcripts was conducted independently by the same research staff responsible for moderating the groups and then cross-checked to assess for consistency.

In order to produce a relaxed atmosphere conducive to open discussion it is usually desirable for focus groups to be as homogeneous as possible in socio-demographic and/or other characteristics (Parasuraman, 1991). It is also important to quota-sample sub-groups which represent relevant positions on the issues being researched so that any differences between these sub-groups can be identified (Atkin and Freimuth, 1989). Taking into account these requirements the sample incorporated the following quota variables: gender, age, socio-economic group and place of residence (see Table 2).

\section{TABLE 2 ABOUT HERE}

The research sought to sample a sub-population whose age, socio-economic status and lifestyle placed them at elevated risk to cancer. As well as the socio-economic variables described in Table 2, participants were recruited as tobacco smokers (10 or more cigarettes per day), drinking alcohol in excess of the recommended weekly limit (14 units for women and 21 units for men) and eating less than the recommended five portions of fruit and vegetables per day. Participants were recruited from four health board areas in west-central Scotland, a region with high rates of deprivation and higher than average incidence of cancer.

Participants were recruited door-to-door by professional interviewers using a structured recruitment questionnaire to ensure they met the socio-demographic and behavioural selection criteria. Confidentiality was ensured in accordance with statutory requirements and each participant was offered a small fixed financial incentive to cover costs incurred in taking part in the research. All participants were informed that the taking part would involve discussing cancer and were given the opportunity to opt out of the research at any 
point. In order to deal with any concerns raised by the research, relevant information materials and sources of support and advice were made available at the end of each discussion.

\section{$\underline{\text { Results }}$}

The results presented here relate to the study population's general perceptions of the disease and of early intervention concepts, and are organised into four discrete study themes: perceptions and attitudes to cancer, perceptions and attitudes to early detection, response to early intervention campaigns, and self-referral process and sources of advice. Key findings are illustrated using verbatim quotations drawn from voice-transcripts. These have been anonymised where necessary and key respondent characteristics described in terms of gender (male, female), age group (40 to 55 years and 56 to 70 years), socio-economic group (C1C2 or middle-income groups and DE or lower income groups) and area of residence (rural community and urban community).

\section{Perceptions and Attitudes to Cancer}

Cancer was regarded as a common disease which could affect all sectors of Scottish society. The majority of participants knew of at least one person in their social network, typically an elderly relative or family friend, who had cancer or who had died from the disease. A smaller number had personal experience of the disease.

Despite its high incidence, participants described a general reluctance to discuss cancer which they equated with fear and medical sciences inability to find a breakthrough cure for the disease. Terms such as 'taboo', 'covered-up' and 'pushed under the carpet' were frequently used when describing people's willingness to discuss the disease. Interestingly, this apparent reluctance was less evident in the discussion groups with participants keen to use the opportunity to voice their opinions and describe their experiences of the disease. There was also a widely held belief that people were more prepared to talk about the disease than had been the case in the past, with some regarding this as part of a general trend towards a greater openness about difficult social issues.

"When my mum had it [cancer] it was taboo. They didn't talk about it years ago. I found out through my Dad when I had it, 'Oh, your Mum had cancer'." (Female, 40-55, C1C2, Urban)

"You never heard about it years and years ago. It's the same as other illnesses like Alzheimer's - they were just put under the carpet."

"A lot of things are out in the open compared to what they used to be."

(Female, 56-70, DE, Rural) 
The influence of popular media and in particular television programmes such as soap operas, chat shows, hospital dramas and the TV news appears to have a normalising effect, and to reflect peoples growing willingness to confront the disease.

"Nowadays it's more to the forefront, isn't it? It's there. Every time you look at a paper someone has got it"

(Male, 40-55, C1C2, Urban)

"Now you see it on the telly all the time. It seems like everyday they have got a new cure."

"If somebody famous died with anything like bowel cancer, if it goes on the news, everybody will be in the pub ... 'Did you hear so-and-so died of bowel cancer?' ... Everybody would be talking about it."

(Male, 55-70, DE, Urban)

However, despite these developments the topic continues to arouse strong emotions. While some acknowledged that many cancers were treatable and could be cured, there was a considerable degree of fatalism surrounding attitudes to the disease, more so among older participants.

"None of us know and it's the roll of the dice for every single one of us. If we are going to get it we get it. There's nothing in this world we can do"

(Male, 40-55, C1C2, Rural)

"I just think if it's in your blood it's going to come out. It's there."

(Female, 56-70, DE, Rural)

Fatalism and a reluctance to confront cancer were also important to how participants related known risk factors to their chances of contracting the disease. This was most apparent with smoking, and to a lesser extent diet. Alcohol was much less likely to be linked with cancer. Most noteworthy was participants' vociferous defence of their smoking behaviour.

"Everything's blamed on smoking - and it's definitely not true."

"I've known an awful lot of my family that's died of cancer that's never smoked in their life."

(Female, 56-70, C1C2, Urban)

"All my family were smokers. Not one of them died of cancer." 
(Male, 40-55, C1C2, Rural)

Some participants found solace in the fact that many people who die from cancer have apparently healthy lifestyles and appear fit and healthy - or as one heavy smoker stated, "that's what gives the likes of me a bit of hope." These observations were frequently used to rationalise the decision to continue smoking, with some also arguing that stopping smoking at their time in life could actually prove harmful to health.

"I think it would be a bigger shock to your system if you'd smoked for years and then all of a sudden stopped."

(Female, 56-70, C1C2, Urban)

"I know folk that's stopped smoking and they've been not well since. I think the nicotine keeps the germs away."

(Male, 40-55, C1C2, Rural)

Taken together these findings indicate that given the fears and limited readiness to change, great awareness and sensitivity is required when developing interventions which seek to engage people in the prevention of cancer.

\section{Perceptions and Attitudes to Early Detection}

Early detection was a familiar concept, more so amongst women, many of whom were regular participants in mass screening programmes. It was also widely recognised that early detection and treatment improved chances of survival, and that the individual had the potential to affect their own health by presenting early if they experienced suspicious symptoms.

"If you leave it too late, it's curtains anyway. They tell you nowadays if they catch it in time they can do wonders."

(Male, 55-70, DE, Urban)

"Eighty to ninety percent if they get it early enough, they can treat it. It's getting it early enough."

"I think every day you stay alive is another day they are looking for a cure."

(Male, 40-55, C1C2, Rural)

Whilst self-awareness and self-examination were regarded as important pre-conditions to early detection, knowledge of cancer signs and symptoms was extremely variable. Signs commonly mentioned included 'lumps' (particularly in the breasts and testis), 'bleeding or unusual discharge' (particularly in the stool or phlegm), 'weight loss' and 'tiredness and malaise'. There was also an appreciation that different cancers, or cancers located in different parts of the body were likely to present with different symptoms, although 
understanding of symptom specificity and of the association between symptoms and cancer stage were extremely limited.

Fear of cancer and high levels of mortality associated with the disease were a recurring theme in these discussions and had a negative impact on peoples willingness to consider self-detection strategies and to seek the opinion of a health professional or to discuss symptoms with family or friends. This pattern of response was expressed in different ways. Women were more likely to recognise and verbalise these fears:

"Everything would put me off. I'd be petrified."

"You just suffer it. It's the only chance you've got."

(Female, 56-70, DE, Rural)

"You're frightened what they're going to tell you."

"I think it's the unknown."

"... Your mind starts to run riot a bit - you think of all the things that's connected."

(Female, 56-70, C1C2, Urban)

Men, on the other hand, were more inclined to deny these fears, which in some instances led them to adopt extreme and entrenched positions on the issue.

"I believe that once you've got cancer you've got it. I've known people who have had cancer and not one of them is this day living. Nothing can be done."

(Male, 40-55, C1C2, Rural)

So whilst the benefits of early presentation and diagnosis were widely accepted at a rational and objective level, when viewed at a personal level, the strong emotions which cancer can arouse appeared to act as a barrier to confronting the issue.

"They can treat it, if you are quick enough in catching it yourself."

"It's having the guts to go up to the doctor and say, 'Look, I think this is wrong with me'."

(Male, 40-55, C1C2, Rural)

Closer analysis revealed this was not a simple dichotomy as the same individual could hold both views simultaneously, their expression being largely dependent upon context. For example, in the period of just a few minutes one participant said she would advise "anyone" to go forward with suspicious symptoms:

"I feel if you go soon enough there is the possibility that they can do something. If you let it go, by the time you do go it's past the stage for help."

(Female, 56-70, C1C2, Urban) 
But when invited to consider how she might react when confronted with the same symptoms she expressed an apparently contradictory view:

"When your time comes you'll go, no matter what ... I'd rather not know."

(Female, 56-70, C1C2, Urban)

Importantly, participants were sometimes quite prepared to acknowledge these contradictory positions. For example, a similar pattern was observed when the same group of participants was probed on the role of lay-support and entry to the health care system.

"I would advise them [friends] to go to the doctor but when it comes to yourself, it's different."

(Female, 56-70, C1C2, Urban)

These apparently contradictory behaviours would appear to be explained by participant's inability to deal with risk and uncertainty, with medical intervention failing to guarantee the positive health outcomes sought.

\section{Response to Early Intervention Campaigns}

In keeping with peoples' objective evaluation of the benefits of early detection, participants were generally supportive of interventions designed to promote secondary prevention, although understanding of the concept and its relationship with curative procedures was sometimes limited.

"Early detection - I'm a great believer in that. Prevention is better than cure."

(Male, 40-55, C1C2, Rural)

Participants also supported the principle of patient education and raising public awareness of the early signs and symptoms of cancer. This was a familiar idea with some recalling having seen information posters in health care premises and patient waiting areas raising awareness of specific cancers and their signs and symptoms.

"I think in a lot of the surgeries now have signs up saying if there is any change in your bowel habits get it seen to.

Make sure it is nothing."

(Male, 55-70, C1C2, Urban)

Support for these campaigns was premised on the belief that the individual could benefit in one of two ways: either from peace of mind from knowing that they were cancer free 
or, more significantly, from improved chances of survival where the cancer is diagnosed and treated early.

"If it is cancer you get it early and they can deal with it."

(Male, 40-55, C1C2, Rural)

"... there is always a strong possibility that it will be clear.

It puts your mind at ease."

(Female, 56-70, C1C2, Urban)

However, consistent with earlier findings, when participants considered how they were likely to react to this type of message if subject to the symptoms highlighted at time of exposure, responses were characterised by feelings of angst and uncertainty, and led some to question the likely impact on demand for health services.

"I think that would worry people."

"It would worry a lot of people, but in another sense there would be a lot of people ignorant of the facts altogether."

"But you're as well being ignorant."

“Not really."

"I'll stay the way I am."

"I think a lot of people would be oh..."

"I think there are quite a few that could make people panic for nothing."

(Female, 56-70, C1C2, Urban)

"I think the doctors might find they are suddenly being inundated by people taking up their time because they are panicking. I think doctors have got too much on their plate as it is."

(Female, 56-70, C1C2, Urban)

Cultural factors appeared to have an important role in determining how the different genders were likely to respond to this kind of health information, with female participants showing a greater propensity to seek professional advice than male participants. This difference appeared to reflect women's greater familiarity and use of primary health care services through, for example, participation in mass screening programmes and responsibility for children's health care. In contrast men, particularly men living in rural areas, were less inclined to acknowledge health problems, and where illness was 
recognised they generally preferred to self-treat than to consult a doctor or health professional.

"A man's more likely to sit there for months and say, 'It'll go away', whereas a woman will go down to a Well Woman clinic or something like that - there are eight of us here, if you go round the eight of us and say how many of you would go down to a Well Man clinic we'd be looking at each other to see which one is the poof."

"It's not within their psyche, especially in the west of Scotland."

"It’s their make-up."

"If you find a wee lump, like the bloke was saying, you say,

'It'll go away'. You ignore it ... and before you ken where you are it is the size of a football."

(Male, 40-55, C1C2, Rural)

"Men are hopeless, you have to push them along the road."

"I think they feel they shouldn't have anything wrong with them."

"They don't like talking about certain things. I think they are frightened. They don't like facing up to [things]."

"I don't think they can cope with the result if it was bad.

Men cannae cope the same as women."

"They wouldn't let on, I don't think."

(Female, 56-70, C1C2, Urban)

"If you stay in a rural community like this, you tend to find things you do yourself more than anything else. If you've got a bit of toothache you fix it yourself. I think that is the same with illnesses as well. If you end up with the cold you're not at the doctor's every two minutes. Down here you just don't do that. You just sort it yourself. It's not like a big city where people are at the doctors every two minutes." 
(Male, 40-55, C1C2, Rural)

Patient education campaigns designed to raise awareness of the early signs of cancer therefore commanded wide public support, although taking into account peoples fears associated with the disease in campaign development is critical to their effectiveness.

\section{Self-referral Process and Sources of Advice}

A variety of sources emerged as first point of contact for advice about suspicious symptoms. These included primary care professionals, helplines and lay sources such as partners and close friends. The 'family doctor' or 'GP' (general medical practitioner) was widely regarded as the most likely point of contact with the health care system. Consistent with earlier findings, women tended to consult with their GP more regularly than men. This was largely the consequence of family health care responsibilities and availability of women's health care services.

"I hardly ever go to the doctor. I'm not saying I never go but I don't go a lot." (Male, 40-55, DE, Urban)

Readiness to present to a GP was influenced by a number of factors. A common theme was people's expectation of how the doctor is likely to respond to the patient, and in particular patient perceptions of whether the GP is likely to take the patients concerns seriously. Importantly, there was a widely held belief that response to the same symptom could vary markedly between GPs:

"It depends on the doctor. Some of them would say, 'Right, I'll get that seen to right away, get you seen to by a specialist'. Other ones would turn round and say, 'We'll gie you these tablets, try these, in a week's time come back and let me know if it's still the same', I think it's an awful lot to do with your GP."

(Female, 56-70, C1C2, Urban)

A number of factors appeared to mediate these expectations: the nature of the symptom (whether it was perceived by the patient as being potentially minor); the reputation of the GP (patient perceptions of previous consultation outcomes); and the patient-doctor relationship (patient ability to communicate symptoms to the GP).

"You don't want to turn round after a day and say, 'I'd better go to the doctor, I've got a mouth ulcer or this whiteness'... You go there and he pooh-poohs you - you're wasting that guy's time as well ... You'd think twice about 
it, wouldn't you? You'd want to go with something serious."

(Male, 40-55, DE, Urban)

"We've got seven GPs in our surgery and my GP is the type if you go in with a sore finger or a sore foot or something - 'It's smoking' ... But if you go to another one, he's not so bad."

(Female, 56-70, C1C2, Urban)

"My doctor would just sit and stare at the computer. I just cannae talk to him. ... You just go in and you don' tell him what you are exactly down for. You make an excuse just to get back out of there. "

(Female, 40-55, C2C2, Urban)

Other factors to emerge included, GP gender (although the significance of gender varied according to the sensitivity of the symptom) and appointment process, where long delays provided some participants with a justification for not presenting and were seen to conflict with messages urging early presentation.

"Phoning your doctor and getting told you'll get an appointment a week later. That is a put off right away."

(Female, 40-55, C1C2, Urban)

Financial cost did not emerge as a barrier to accessing GP support as medical care is provided free at point of need with this study population, although the same is not the case for dental care. It was generally accepted that routine dental checks could increase the chances of detecting oral cancers. However, some of those on lower incomes were deterred from attending for dental checks on cost grounds.

"I don't go to the dentist."

“I couldn't afford it anyway."

"Cost again, keeps you away from that 6-monthly check." (Male, 40-55, DE, Urban)

Whilst general medical practitioners were regarded as the main source of professional advice for any concerns relating to cancer, other primary care professionals, most notably community pharmacists and primary care nursing staff, could be consulted on an opportunistic basis. It was suggested that other members of the primary health care team had a role to play in raising awareness of potential serious symptoms and directing patients presenting with such symptoms to seek advice from their GP.

"They're quite good at giving out advice (pharmacists)." 
"Especially for something like this (mouth ulcers) - for everyday things."

"I'd go to the doctor (about cancer)."

"If I had an ulcer or something I might speak to the pharmacist."

(Female, 56-70, DE, Rural)

Lay sources of advice also emerged as an important part of the consultation process. Participants, particularly female participants cite lay networks as an important source of reassurance prior to seeking professional advice, in some instances providing additional emotional support when attending for appointments. Lay sources also appeared to have a critical role in encouraging men to confront their symptoms and to seek professional advice.

"I think if you had these symptoms you would go and talk to another woman about bowel cancer ... before you would go to your doctor."

"But deep down in your mind you know you have got to go."

"You're frightened in case you are wasting the doctor's time."

"Whereas if it is a friend and they say, 'No, I think you should go to the doctor'."

(Female, 56-70, C1C2, Urban)

"If you have got close friends they would advise you to go to the doctor and they would probably say, 'If you are nervous I'll come with you'."

(Female, 56-70, C1C2, Urban)

"My brother-in-law had trouble with the waterworks and he was reluctant to go to the doctor. I talked him into going. The doctor examined him and sent him to the hospital. He had an infection. Antibiotics cleared it up within a week and he had suffered for months."

(Male, 55-70, C1C2, Urban)

"Any time my husband goes to the doctor I have to drag him there, literally." 
"I think men are more inclined to push it to the back of their mind and not even say anything to you until the last resort."

(Female, 56-70, C1C2, Urban)

In addition to relying on social networks for support participants also had strong ties with their local communities and some were regular participants in local events and activities. Limited mobility and a community centred ethos meant that some rarely ventured out with their local area.

\section{$\underline{\text { Discussion }}$}

The existing evidence-base highlights a range of modifiable factors which can influence the decision to self-refer. These include fear (Nosarti et al. 2000), a failure to recognise the potential significance of the signs (Nosarti et al. 2000, Burgess et al. 2001, Crossland and James 1995) and consulting with and support from family and friends (Burgess et al. 1998). In addition, the patient-physician relationship and anticipated response to presentation has also been found to play an important role (Burgess et al. 2001, Davey et al. 1999, Bish et al. 2005).

Our research findings confirm the importance of many of these factors and illuminates how they manifest themselves amongst a specific at-risk population. Whilst cancer was seen by many as a relatively common condition with wider media coverage of the disease encouraging more open discussion, the disease continues to arouse considerable fear, leading to what might be regarded as irrational responses. For example, whilst it was widely acknowledged that treatments were improving there was a reluctance to confront the disease.

There is also evidence that these factors are responsible for limiting help-seeking behaviour, particularly amongst men. Whilst there is considerable recognition of the concept and benefits of early detection and generalised knowledge of signs of the disease, there is a reluctance to act on the symptoms. As a consequence, many are more willing to advise others to present with symptoms. This suggests that information campaigns targeting key opinion formers within patients' lay networks could have an important persuasive role. Older respondents living in deprived communities of interest to the study were particularly reliant upon local community networks for support and advice, whilst women were more inclined to discuss symptoms with family and friends, and to have an influential role in encouraging men to seek medical advice.

The general medical practitioner or GP emerged as a key source of professional advice for members of the target population and was the main point of entry into the health care system. However, readiness to self-refer was mediated by past experiences and anticipated response by the GP, with concerns that s/he would dismiss potentially trivial symptoms responsible for some delays. Importantly, the decision of the GP to invite the patient to make a repeat appointment if the symptom persisted can be interpreted by some 
patients as evidence of wasting the doctors' time. Delays due to structural factors, such as the expectation of having to wait for a week or more for an appointment, are interpreted in a similar manner. Other primary care professionals such as community pharmacists and practices nurses were seen as more approachable sources of opportunistic advice and can have an important role to play in encouraging patients to refer to their GP.

Whilst there is an abundance of information on public awareness and attitudes towards those cancers where mass screening is undertaken, most notably breast and cervical cancer, for other less prominent cancers there tends to be a reliance upon retrospective data from patients diagnosed as having cancer. As a consequence, these studies have tended to focus upon patient pathways and treatment process. This study provides insights into the decision-making processes involved in presenting with symptoms of cancer amongst a population at risk of cancer due to age, socio-economic and lifestyle factors, and highlights modifiable factors which can facilitate and hinder help-seeking behaviour. The 'real world' context of the individual as participant as opposed to patient, illuminates the milieu in which the decision to seek help is taken: the embedded nature of fear of cancer and the link with death; the relatively superficial knowledge of signs of cancer and their relationship with 'normal' changes; the mix of rational, although at times imperfect, understanding of benefits of early detection, and emotional responses that discourage personal engagement with the issue. The study also gives insights into how social structures and relationships can influence the decision to present: the impact of previous experience of contact with physicians and anticipated response; the value of other primary care professionals in providing opportunistic advice; and the importance of family, lay support networks and community organisations.

These insights have wide-ranging implications for the development of early detection interventions designed to promote self-referral. To ensure appropriate and timely presentation careful consideration needs to be given to the content and tone of the early detection message. Messages need to be grounded in an understanding of how at-risk populations currently make sense of the cancer symptoms being targeted and how these can be differentiated from 'normal' changes. In addition, they also need to build confidence in the benefits of early detection and to minimise arousing unnecessary fear. Similarly, attention needs to be given to the use of social structures to reinforce communication of the early detection message. Primary care professionals not only need to be aware of the early signs, but also need to be cognisant of people's fears and anxieties in coming forward and how they are likely to interpret diagnostic decisions and advice. The findings from this study also endorse the use of lay and family networks in supporting and encouraging early presentation, particularly when targeting men.

\section{Limitations}

Whilst the qualitative methods employed provided the necessary scope and flexibility to identify and explore the relationship between individual level factors responsible for influencing self-referral behaviour, the small sample size required by this research procedure means it does not represent the entire study population. In addition, the investigators also recognise that the results presented here related to cancer as a generic 
disease and that attitudes and behaviours will vary according to type of cancer. In view of these issues, the generalisability of the results arise not from the sample's representativeness, but from the characteristics of the factors identified and their value to the development of specific measures and assessment with larger samples and across a range of cancers.

\section{$\underline{\text { Acknowledgements }}$}

This study formed part of the West of Scotland Cancer Awareness Project funded by the New Opportunities Fund.

\section{$\underline{\text { References }}$}

Anderson BI and Cacioppo JT (1995). "Delay in seeking a cancer diagnosis: Delay stages and psychological comparison processed”. British Journal of Social Psychology, 34(part1): 33-52.

Atkin CK and Freimuth V (1989). "Formative evaluation research in campaign design". In RE Rice and CK Atkin (eds), Public Communication Campaigns, $2^{\text {nd }}$ edition (pp131150). Newbury Park, CA: Sage Publications.

Beeker C; Kraft JM; Southwell BG; Jorgensen CM (2000) "Colorectal cancer screening in older men and women: qualitative research findings and implications for intervention", Journal of Community Health, 25(3): 263-278.

Bish A, Ramirez A, Burgess C and Hunter M (2005). "Understanding why women delay in seeking help for breast cancer symptoms”. J Psychosom Res, 58(4): 321-326.

Bloom JR (1994). “Early detection of cancer”. Cancer, 74 (suppl 4): 1464-1473.

Box V (1984). “Cancer: myths and misconceptions”. Journal of the Royal Society of Health, 104(5):161-166.

Braveman P and Tarimo E (1996). "Health screening development and equity”. Journal of Public Health Policy, 17(1): 14-27.

Burgess CC, Hunter MS and Ramirez AJ (2001). “A qualitative study of delay among women reporting symptoms of breast cancer”, British Journal of General Practice, 51: 967-971.

Burgess CC, Ramirez AJ, Richards MA, and Love SB (1998). "Who and what influences delayed presentation in breast cancer?” British Journal of Cancer, 77(8): 1343-1348. 
CDCP (2002). Guidance for Comprehensive Cancer Control Planning, Volume 1:

Guidelines. Centers for Disease Control and Prevention, Atlanta, March 2002.

Crossland A and James R (1995). "Rectal bleeding: prevalence and consultation behaviour”. BMJ, 311(7003): 486-488.

Davey C, Austoker A and Macleod U (1999). Reducing Inequalities in Breast Cancer: A Guide for Primary Care. London: CRC.

Duyn A, Van Eijkeren M, Kenter G, Zwinderman K and Ansink A (2002). "Recurrent cervical cancer: detection and prognosis”. Acta Obstetricia Et Gynecologica Scandinavia, 81(8): 759-763.

Eiser JR, Pendry L, Greaves CJ, Melia J, Harland C and Moss S (2000). "Is targeted early detection for melanoma feasible? Self-assessment of risk and attitudes to screening”. Journal of Medical Screening, 7(4): 199-202.

Nosarti C, Crayford T, Roberts JV, Elias E, McKenzie K and David AS (2000). "Delays in presentation of symptomatic referrals to a breast clinic: patient and systems factors".

British Journal of Cancer, 82, 742-748.

Parasuraman A (1991). Marketing Research. Addison Wesley.

Quinn M, Wood H, Rowan S and Cooper N (2005).Chapter 2: “Geographical patterns in cancer in the UK and Ireland". In Quinn M, Wood H, Rowan S \& Cooper N (eds.) Cancer Atlas of the United Kingdom and Ireland 1991-2000. Studies on Medical and Population Subjects No. 68. London: Office for National Statistics. ISBN:1403996458.

Ristvedt SL and Trinkaus KM (2005). "Psychological factors related to delay in consultation for cancer symptoms”. Psycho-Oncology, 14(5): 339-350.

Truman BI, Gebbie KM, Lawrence RS, Evans CA, Mahan CS and Zaza S (2000) "Developing the guide to community preventive services - overview and rationale", American Journal of Preventive Medicine, 2000;18(1S): 18-26.

Wolf RL, Zybert P, Brouse CH, Neugut AI, Shea S, Gibson G, Lantiua RA, Basch CE (2001). "Knowledge, beliefs, and barriers relevant to colorectal cancer screening in an urban population: A pilot study”. Family Community Health, 24(3): 34-47.

\section{Table 1: Discussion Themes}

\section{Theme 1 - Cancer Awareness}

Awareness and perceptions of cancer, including: signs and symptoms, seriousness and survival rates, perceptions of who is at risk, and awareness and understanding, if any, of measures to help detect and prevent cancer.

Theme 2 - Presentation with Potential Symptoms of Cancer 
Preparedness to undertake regular self-examination and to present to primary care or other health care providers with suspicious signs and symptoms. Particular emphasis was placed on examining the social and structural barriers (perceived and real) which could deter self-referral.

Theme 3 - Health Information Campaigns

Attitudes towards, and experience of, health information campaigns: factors which enhance response with regards to message, channel, language and tone of communication. A selection of cancer awareness materials were used to stimulate discussion and to examine alternative message and media formats.

Table 2: Sample

\begin{tabular}{|c|c|c|c|c|}
\hline Group No & Gender & Age & Socio-economic Group* & Place of Residence \\
\hline 1 & Male & $40-55$ & C1C2 & Rural \\
2 & Female & $56-70$ & C1C2 & Urban \\
3 & Male & $56-70$ & DE & Urban \\
4 & Female & $40-55$ & DE & Urban \\
5 & Male & $40-55$ & DE & Urban \\
6 & Female & $56-70$ & DE & Rural \\
7 & Male & $56-70$ & C1C2 & Urban \\
8 & Female & $40-55$ & C1C2 & Urban \\
\hline
\end{tabular}

* C1 \& C1 represent middle income groups and D \& E low income groups 